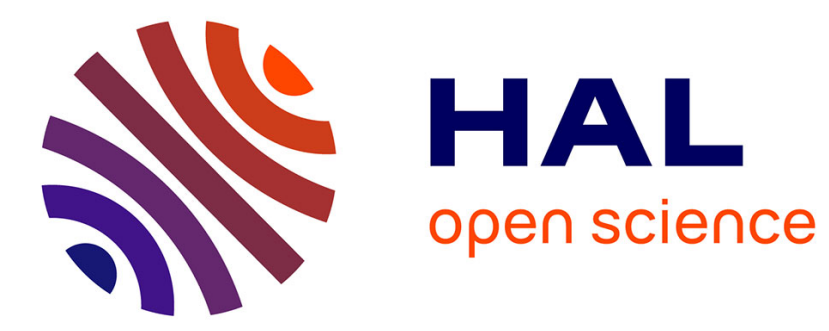

\title{
Acquisition de la propreté et dyschésie psychologique: thérapie comportementale chez le jeune enfant
}

Céline Clément

\section{To cite this version:}

Céline Clément. Acquisition de la propreté et dyschésie psychologique: thérapie comportementale chez le jeune enfant. Journal de Thérapie Comportementale et Cognitive, 2014, 24 (2), pp.47-52. 10.1016/j.jtcc.2014.04.001 . hal-01099616

\section{HAL Id: hal-01099616 https://hal.science/hal-01099616}

Submitted on 4 Jan 2015

HAL is a multi-disciplinary open access archive for the deposit and dissemination of scientific research documents, whether they are published or not. The documents may come from teaching and research institutions in France or abroad, or from public or private research centers.
L'archive ouverte pluridisciplinaire HAL, est destinée au dépôt et à la diffusion de documents scientifiques de niveau recherche, publiés ou non, émanant des établissements d'enseignement et de recherche français ou étrangers, des laboratoires publics ou privés. 
Nom de l'auteur : Céline Clément

Titre : Acquisition de la propreté et dyschésie psychologique : thérapie comportementale chez le jeune enfant

Title: Toilet training and psychological dyschezia: Behavioral therapy in young children

E-mail : celine.clement@unistra.fr

\section{Adresse :}

École Supérieure du Professorat et de l'Éducation - Laboratoire Interuniversitaire des Sciences de l'Éducation et de la Communication,

Université de Strasbourg

141 avenue de Colmar, 67100 Strasbourg

\section{Résumé}

L'acquisition de la propreté chez l'enfant est une étape importante vers l'autonomie. Si l'énurésie et l'encoprésie sont les difficultés les plus fréquemment décrites pour ce qui a trait au contrôle sphinctérien, la difficulté d'exonération des selles est peu documentée. Récemment, la dyschésie psychologique et sa prise en charge dans un abord cognitivocomportemental ont été décrites. Le présent article vise à présenter le déroulement de la thérapie telle que nous l'avons conçue et réalisée chez une jeune enfant pour laquelle les cognitions étaient peu accessibles. L'utilisation conjointe du façonnage, en utilisant des renforçateurs secondaires, puis primaires, et la suppression de tout accès aux couches-culottes s'est avérée un succès. D'autres cas cliniques sont à décrire afin de fournir aux cliniciens des outils appropriés pour l'accompagnement de l'enfant et de sa famille.

\section{Mots clés : Dyschésie psychologique, TCC, Enfant, Cas clinique, Contrôle sphinctérien}

\section{Summary}

Toilet training in children is an important step toward autonomy. Enuresis and encopresis are the most frequent difficulties met in sphincter control. Difficulties in evacuating stools are 
less described. Dyschezia is another kind of sphincter control disorder: the incapacity or difficulty in evacuating stools naturally. The concept of psychological dyschezia - dyschezia with a psychological etiology-has recently been developed. It is defined as either the incapacity or the difficulty in evacuating stools (difficult or slow evacuation requiring prolonged efforts), or as the retention of stools without a biological or functional anomaly. The present paper is a case study of a 4-year-old little girl who presents a symptomatology allowing the diagnosis of psychological dyschezia. This little girl, with no physiological problems or other psychological symptomatology, was only able to defecate in a diaper and refused to sit on the toilet. A functional analysis was conducted: (1) anger was shown when the girl was asked to sit on the toilet; (2) the cognition was that "it's easier to do it when I'm standing"; (3) the reinforcer was the use of diapers. The intervention consisted in successive reinforcement conditions. Although the reinforcer was the diaper, the choice was made to maintain access in order to avoid constipation. At first, the focus was on shaping the behavior "bending the knees while standing on a small footstool" while maintaining access to diapers. Using a token economy, this shaping phase was successful in a few days. In the second condition, the objective was to reinforce the behavior "pushing on the toilet" while gaining access to a diaper after pushing 2 or 3 times. The reinforcers used were a token and a special candy. This condition was unsuccessful. In a third and last condition, access to the diapers was completely removed and the special candy was maintained. This condition was a complete success in one trial. The concept of psychological dyschezia was useful to propose an intervention based on cognitive and behavior therapy for this 4-year-old girl. Nevertheless, based on the fact that the child was able to defecate with no difficulties in a diaper and to sit on the toilet to urinate, our intervention was centered on the behavior "sitting on the toilet to defecate" instead of the cognition. Other cases studies have to be described in order to develop guidelines for clinicians who are confronted to dyschezia with a psychological etiology.

\section{Keywords : Psychological dyschezia, CBT, Child, Single case, Sphincter control}




\section{Introduction}

L'acquisition de la propreté chez le jeune enfant est un apprentissage important dans nos sociétés. Si en France il ne conditionne pas l'entrée en maternelle à 2 ou 3 ans, il s'impose comme une nécessité pour de nombreux parents. Cet apprentissage est en effet une étape importante de l'autonomie de l'enfant, lui permettant entre autres de séjourner seul en dehors du domicile chez des amis ou de la famille et de mener des activités périscolaires.

Cet apprentissage a donc une incidence importante sur le développement social, cognitif et émotionnel de l'enfant [1]. De plus, même si cet aspect est peu abordé dans la littérature scientifique, celle-ci se centrant davantage sur l'aspect médical, l'incidence des difficultés d'acquisition de la propreté est importante. Les forums de discussion regorgent de sujets traitant cette question, les parents étant dans la crainte d'un retard d'acquisition ou se déclarant impuissants après « avoir tout essayé » et regrettant leurs accès de colère. Moins fréquemment la question du coût engendré par l'achat des couches jetables, soit plusieurs milliers d'euros de 0 à 36 mois, peut être évoquée.

Le rythme de l'apprentissage de la propreté est bien connu [1] et en général, dans nos sociétés industrialisées, à trois ans la propreté nocturne et diurne sont acquises. Cependant il n'est pas rare qu'émergent des difficultés dans cet apprentissage. Au-delà de difficultés ponctuelles, les enfants peuvent présenter de véritables troubles du contrôle sphinctérien, tels que définis par le DSM-IV [2]. Ils apparaissent durant la petite ou la seconde enfance. Les données les plus fréquentes portent sur l'énurésie et l'encoprésie [3], qui sont une miction ou une émission fécale involontaire et persistante dans des endroits non appropriés, à un âge auquel l'enfant devrait être continent. Ainsi il est rapporté une fréquence d'énurésie nocturne de $25 \%$ à 4 ans, $10 \%$ à 6 ans, $5 \%$ à 10 ans et $1 \%$ à 15 ans [4]. La constipation est une autre difficulté qui serait présente chez un tiers des enfants des pays occidentaux [5].

Parmi les différentes formes de constipation, on retrouve la dyschésie qui se caractérise et se différencie des autres formes de constipation par le fait que les selles sont normales ou molles et non pas dures ; ceci est particulièrement observé chez l'enfant [7]. Deux types de causes à la dyschésie sont généralement décrites : les causes organiques (p.ex. fissure anale, tumeurs rectales, sclérose en plaque) [8] et les causes fonctionnelles (p.ex. dysfonctionnement des sphincters de 1'anus) [9]. Récemment Boudoukha a décrit une troisième forme de dyschésie, la dyschésie psychologique [1]. Il la définit comme l'incapacité ou la difficulté à exonérer ses selles (exonération difficile ou lente nécessitant des efforts prolongés) ou encore la rétention de la selle en l'absence d'une atteinte organique ou fonctionnelle actuelle. S'appuyant sur la 
forme des critères diagnostics de la Classification Internationale des Maladies ou du DSM, l'auteur présente une série de critères diagnostics (tableau 1).

Tableau 1 Proposition de critères diagnostiques pour la dyschésie psychologique chez l'enfant.

Diagnostic criteria for child psychological dyschezia (adapted from Boudoukha, 2013).

Difficulté de l'exonération des matières fécales avec au moins l'un des symptômes suivants :

- l'exonération nécessite des efforts de poussées prolongées et provoque des plaintes chez l'enfant

- la rétention des selles est totale en dehors de la présence d'un tiers (la mère ou le père)

- pour permettre l'exonération l'enfant ne peut pas aller à la selle dans un endroit approprié (toilette, pot...)

- l'enfant doit porter sa couche pour parvenir à l'exonération

Cette difficulté ou incapacité n'est pas liée à une atteinte organique ou fonctionnelle actuelle (au moment où l'enfant est rencontré, il ne rapporte pas une atteinte même s'il est possible que par le passé, il ait pu souffrir d'une telle atteinte)

Le comportement est cliniquement significatif, soit par sa présence depuis au moins trois mois consécutifs, soit par la présence d'une souffrance cliniquement significative ou d'une altération du fonctionnement social, scolaire, ou dans d'autres domaines importants

L'enfant a atteint un âge (développement fonctionnel) qui lui permet un contrôle sphinctérien

Le comportement n'est pas dû exclusivement aux effets physiologiques d'un régime alimentaire particulier, d'une substance, ni à une affection médicale générale (fissure anale, dysfonctionnement des sphincters de l'anus, troubles de la statique pelvienne postérieure. . .)

Cette troisième forme de dyschésie semble tout à fait pertinente et utile pour le clinicien. En effet, il n'est pas rare de rencontrer des patients pour lesquels les médecins ne mettent en évidence aucune cause organique ou fonctionnelle aux difficultés à la défécation, y compris chez l'adulte [10]. Dans ces cas, les traitements classiques de la dyschésie (rééducation musculaire et changements diététiques) [11], y compris chez l'enfant, ne sont pas opérationnels.

Cependant les plaintes au sein du foyer ou auprès des pédiatres sur les difficultés de l'acquisition de la propreté sur le plan des selles, ne sont pas rares à un plus jeune âge. En effet comme le rappelle Boudoukha [1], la maîtrise du sphincter anal devrait être acquise à l'âge de 36 mois. Ainsi dans une étude de cas convaincante chez un enfant de 4 ans 1/2, Boudoukha [1] utilise les principes de la thérapie comportementale et cognitive pour la prise 
en charge d'une dyschésie psychologique. Dans ce cas, les difficultés de l'enfant sont apparues lors de l'arrivée à l'école maternelle. La psychothérapie a consisté à la gestion du stress, le déconditionnement de la peur lors de la poussée et l'apprentissage même de la poussée. La psychothérapie a donc été axée sur la psychoéducation et sur la gestion de l'anxiété, classique en Thérapie Comportementale et Cognitive.

Nous proposons ici un cas clinique chez une jeune enfant de 3 ans et 7 mois. À cet âge, l'accès aux cognitions est plus compliqué, ce qui implique d'axer davantage sur le volet comportemental, différenciant ainsi le travail de celui présenté par Boudoukha.

\section{Illustration Clinique}

\section{Anamnèse}

Emma est une petite fille de 3 ans et 7 mois enjouée et vive, fille unique. Ses parents d'une trentaine d'années vivent en couple et sont salariés. Emma a été allaitée jusqu'à l'âge de huit mois et n'a présenté depuis sa naissance qu'un seul épisode de constipation d'une durée de quelques jours. L'entrée dans le langage oral a été relativement tardive (vers 48 mois pour des phrases à trois mots), mais sans problème de compréhension pour son âge de développement. Elle dort seule dans sa chambre depuis l'âge de deux mois, sans difficulté à l'endormissement.

Du point de vue de la symptomatologie anxieuse, on note vers 2 ans 11 mois, suite à un épisode de varicelle, le refus de porter des vêtements avec des boutons (avec une tolérance pour les boutons non visibles) et des remarques adressées à sa mère lui demandant de changer de vêtements si elle porte des vêtements à boutons. Une hypothèse est émise quant à la généralisation de l'anxiété générée par les boutons de varicelle (douloureux et irritants) aux boutons de vêtements. Les parents sont parvenus en plusieurs mois à un retour à la normale sur le port des vêtements en proposant au choix de l'enfant des vêtements avec de plus en plus de boutons ou des boutons plus visibles.

\section{Histoire du trouble}

La propreté diurne - miction - a été acquise sans difficulté particulière au cours de l'été précédent l'entrée à l'école à 2 ans 7 mois, lorsque les couches ont été enlevées en journée, sieste comprise (aucune procédure de renforcement spécifique, économie de jetons ou autres n'a été mise en place, l'enfant étant systématiquement félicitée comme il est d'usage). 
Antérieurement elle fréquentait la crèche deux journées par semaine, le port des couches y était maintenu, de même que lors des déplacements hors du domicile. Le premier mois d'école a donné lieu à trois épisodes d'incontinence urinaire et un épisode d'incontinence fécale, pendant la sieste. Du point de vue de la miction, l'acquisition de la propreté nocturne a suivi de quelques mois l'acquisition de la propreté diurne. Emma urine sur des toilettes d'adulte, sans réducteur (il est mis à disposition mais n'est généralement pas utilisé). Elle accède aux toilettes grâce à un petit escabeau qui lui permet également de reposer les pieds. Cela ne pose aucun problème au domicile ou hors de celui-ci.

Cependant Emma ne fait ses selles, en général le matin avant de sortir, que lorsqu'elle a accès à une couche-culotte qu'elle peut enfiler seule. Elle se met debout dans un coin de la salle de bains pour déféquer rapidement. Les parents ont tenté à plusieurs reprises de supprimer les couches en expliquant à l'enfant, que faire ses selles assise n'était pas plus difficile que debout, que cela éviterait que ses parents ne se fâchent, que ses amis étaient déjà propres, etc. Cependant les parents ont échoué, l'enfant criant et pleurant dès qu'on lui propose de s'asseoir sur les toilettes, réclamant sa couche-culotte. Afin d'éviter des épisodes de constipation et des maux de ventre à l'école où l'enfant retient également ses selles, les parents finissaient par donner accès à une couche-culotte.

On note deux épisodes où les selles ont été faites sans difficulté sans couche-culotte. Le premier dans un parc, en présence d'enfants et d'adultes non loin, le second au jardin dans des toilettes sèches à l'abri des regards.

Le pédiatre ne suspecte aucun élément organique et a incité l'enfant à aller aux toilettes, auquel cas elle lui donnerait une sucette lors de sa prochaine visite. Bien que l'enfant ait répété cette formule à plusieurs reprises, cela n'a pas été efficace. Aucun traitement pharmacologique n'a été proposé par le pédiatre, compte-tenu du fait que les selles sont molles et quasi quotidiennes.

\section{Analyse fonctionnelle}

Afin d'élaborer un plan d'intervention, une analyse fonctionnelle est menée (figure 1). La cognition présentée dans l'analyse fonctionnelle est directement issue des propos tenus par l'enfant, tandis que l'émotion est inférée à partir des comportements présentés par la petite fille lors de la situation problème. 
Figure 1 Analyse fonctionnelle. Functional analysis.

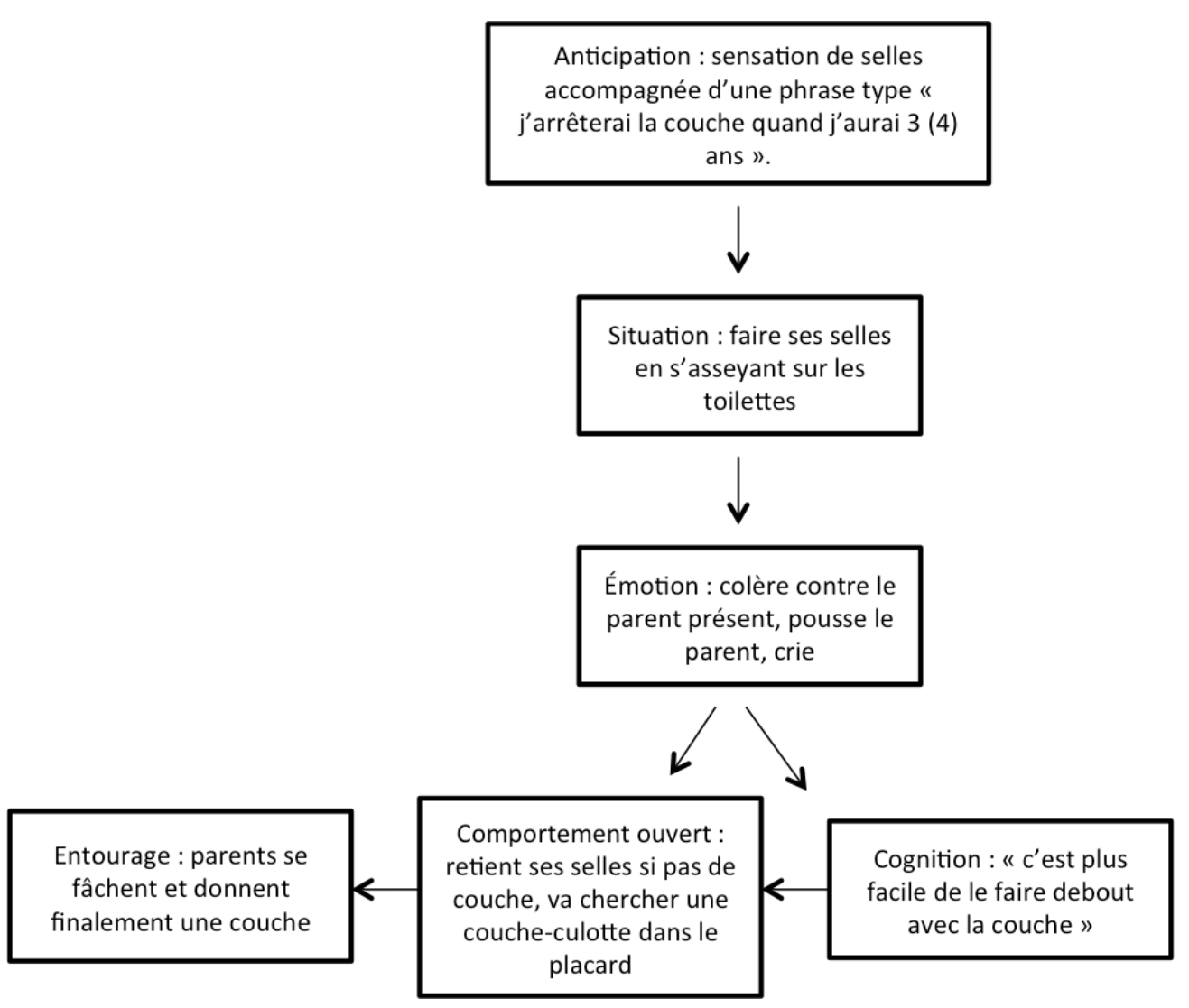

\section{Déroulement de la thérapie}

L'anamnèse, la discussion avec l'enfant et ses parents ne permettent pas d'émettre une hypothèse quant à l'origine du trouble. L'enfant n'évoque pas la peur de la douleur lors de l'exonération, ce aurait pu expliquer son comportement. Elle n'a subi aucune chute connue des toilettes à la maison ou à l'école, et elle parvient à uriner sans difficulté assise. De plus elle pousse correctement lorsqu'elle est debout et sait mimer la poussée assise. Elle est en mesure d'exonérer dans la salle de bains à côté des toilettes et par deux fois accroupie sans couche-culotte à l'extérieur de la maison. La difficulté principale semble donc être le fait de faire ses selles en s'asseyant sur les toilettes, ce qu'on relève sur le cadre situation de l'analyse fonctionnelle (figure 1). En revanche, l'analyse fonctionnelle permet de comprendre le maintien du trouble par l'obtention systématique de la couche-culotte lorsque les parents sont face au comportement problème.

Pour faire face à cette difficulté, tout en souhaitant éviter l'entrée dans une problématique de constipation, il est fait le choix d'utiliser une procédure de façonnage. Celle-ci permet par le 
biais du renforcement positif d'apprendre un nouveau comportement par approximations successives [12]. Ici il s'agit donc de passer de la position «pousser debout» à la position « pousser assise sur les toilettes ». Cette procédure de façonnage est étayée par l'utilisation d'un programme d'économie de jetons [13]. Ce choix est fait parce qu'il n'est pas envisageable que l'enfant fasse ses selles sur les toilettes si elle ne parvient pas à s'y asseoir. D'autre part, compte-tenu de l'âge de l'enfant et des éléments de discussions antérieures rapportés par les parents, un travail sur les cognitions semblait peu approprié. Le programme d'économie de jetons est utilisé car le comportement est un comportement difficile à acquérir pour l'enfant ; il est conçu comme un levier motivationnel.

Le tableau 2 présente les phases et étapes successives de la thérapie. Les phases impliquent principalement des changements soit dans les comportements cibles (plier les genoux vs s'asseoir), soit dans le renforçateur (alimentaire vs tangible). Ce dernier ayant été choisi oralement en concertation avec l'enfant (les bonbons en général et le renforçateur choisi en particulier, ne sont jamais proposés à l'enfant par ses parents en dehors d'une éventuelle apparition du comportement cible). Ces étapes étaient transcrites dans un «cahier des poussées » tel que proposé par Boudoukha [1]. Les contingences (comportement cible et renforçateur conséquent au comportement cible) ont été spécifiées lors de chaque phase à l'enfant.

Si la première phase (se mettre debout sur l'escabeau, puis plier les genoux sur l'escabeau, pour exonérer avec la couche), s'est déroulée sans difficulté majeure, l'étape 2 qui consistait à s'asseoir sur les toilettes avec la couche-culotte ou avec possibilité de la remettre après quelques poussées, s'est avérée un échec. Le choix a été fait de supprimer l'accès aux couches, comme il est recommandé de procéder pour l'acquisition du contrôle de la miction [14]. Dès la première mise en situation, ceci a été une réussite. Emma seule dans les toilettes ayant cherché sans succès les couches habituellement rangées dans un placard, s'est spontanément assise sur les toilettes où elle a fait ses selles rapidement et a exulté de joie. Les jours suivants le comportement s'est maintenu sans difficulté et le renforçateur (une fraise en guimauve) donné systématiquement. Il a été ensuite donné selon un programme à ratio fixe (deux fois sur trois, puis une fois sur deux) pendant trois semaines, puis aléatoirement. La distribution du renforçateur alimentaire a cessé définitivement moins de cinq semaines après la première réussite. L'enfant a réclamé ponctuellement une fraise guimauve pendant une à deux semaines, sans que la non présentation de celle-ci générât la baisse du comportement cible. Aucune difficulté n'est relevée dans les mois qui suivent. 
Tableau 2 : phases de la thérapie.

Successive steps, reinforcers and comments for each intervention phase. The first phase, shaping of bending knees, was a success in 17 days. The second phase, sit on the toilets with access to a diaper after push was unsuccessful. The third phase, sit on the toilets without any access to diapers and access to a special candy was immediately successful.

\begin{tabular}{|c|c|c|c|}
\hline $\begin{array}{l}\text { Phases } \\
\text { de } \\
\text { l'intervention }\end{array}$ & Étapes successives & Renforçateurs & Commentaires \\
\hline \multirow[t]{2}{*}{1} & $\begin{array}{l}\text { Se mettre debout sur le petit } \\
\text { escabeau pour pousser avec } \\
\text { la couche }\end{array}$ & $\begin{array}{l}\text { - « Bonhommes sourires » } \\
\text { en fonction de la réussite. -- } \\
\text { - Cinq « bonhommes } \\
\text { sourires » donnaient la } \\
\text { possibilité d'avoir accès à } \\
\text { des autocollants }\end{array}$ & 5 jours \\
\hline & $\begin{array}{l}\text { Se mettre debout sur le petit } \\
\text { escabeau, avec la couche, et } \\
\text { plier légèrement les genoux } \\
\text { pour effectuer } 1 \text { puis } 2 \text {, puis } \\
3 \text { poussées, avant de } \\
\text { reprendre la position debout } \\
\text { pour exonérer }\end{array}$ & $\begin{array}{l}\text { - « Bonhommes sourires » } \\
\text { en fonction de la réussite. - } \\
\text { - Trois « bonhommes } \\
\text { sourires » donnaient la } \\
\text { possibilité d'avoir accès à } \\
\text { des autocollants }\end{array}$ & 12 jours \\
\hline \multirow[t]{2}{*}{2} & $\begin{array}{l}\text { S'asseoir sur les toilettes et } \\
\text { pousser avec la couche }\end{array}$ & $\begin{array}{l}\text { «Bonhommes sourires » en } \\
\text { fonction de la réussite. }\end{array}$ & $\begin{array}{l}\text { Échec. L’enfant a } \\
\text { réalisé de fausses } \\
\text { poussées (rictus } \\
\text { exagéré sur le } \\
\text { visage ou poussée } \\
\text { très courte). }\end{array}$ \\
\hline & $\begin{array}{l}\text { S'asseoir sur les toilettes et } \\
\text { pousser } 1 \text { fois/ } 2 \text { fois sans } \\
\text { la couche, avec la } \\
\text { possibilité de reprendre la } \\
\text { couche pour finir la } \\
\text { poussée. }\end{array}$ & $\begin{array}{l}\text { Renforçateur alimentaire : } \\
\text { fraise guimauve en cas de } \\
\text { réussite }\end{array}$ & $\begin{array}{l}\text { Échec. Retour au } \\
\text { comportement final } \\
\text { de l'étape } 1\end{array}$ \\
\hline 3 & $\begin{array}{l}\text { S'asseoir sur les toilettes et } \\
\text { exonérer sans la couche / } \\
\text { suppression de l'accès } \\
\text { possible aux couches }\end{array}$ & $\begin{array}{l}\text { Renforçateur alimentaire: } \\
\text { fraise guimauve en cas de } \\
\text { réussite } \\
\text { Ratio fixe } 1 \text { pendant une } \\
\text { semaine, puis ratio fixe } 2 \\
\text { puis } 3 \text {, et enfin ratio } \\
\text { variable }\end{array}$ & $\begin{array}{l}\text { Réussite dès la } \\
\text { première } \\
\text { suppression de } \\
\text { couche et maintien } \\
\text { du comportement }\end{array}$ \\
\hline
\end{tabular}

\section{Discussion}

À partir de la définition de Boudoukha [1] de la dyschésie psychologique de l'enfant et d'un premier cas clinique, nous avons proposé un plan d'intervention pour une petite fille de 3 ans et 7 mois. Compte-tenu des cognitions accessibles et sans difficulté perceptible concernant les poussées elles-mêmes, nous avons choisi d'axer la thérapie sur un versant comportemental. 
Notre choix s'est donc porté sur une procédure de façonnage associant dans un premier temps des renforçateurs tangibles. Si des progrès ont été réalisés, l'assise sur les toilettes avec exonération des selles n'a été possible qu'avec l'utilisation d'un renforçateur alimentaire et la suppression des couches.

La suppression des couches-culottes semble l'élément décisif dans le changement de comportement de l'enfant en phase 3, mais ce changement n'a été possible qu'à partir du moment où la séquence motrice pour l'assise avait été travaillée spécifiquement pour l'exonération des selles (rappelons qu'elle existait pour la miction).

Notre hypothèse est que la présence ou l'absence de couches-culottes à disposition joue comme un stimulus discriminatif, favorisant ou non la présence du comportement cible. Pour l'enfant, avant la dernière phase, l'exonération dans la couche était toujours possible.

Concernant les hypothèses possibles à propos des cognitions, le cas ne permet pas de statuer sur le fait que des changements éventuels dans les cognitions ont précédé ou succédé au changement de comportement. Ce peut être la réussite qui a conduit au changement de cognition ou le changement de cognition qui a précédé le changement du comportement ouvert.

Ceci dit, le changement des cognitions et du comportement ouvert n'a été possible qu'après l'apprentissage de la séquence motrice en phase 1, celle-ci permettant de réduire la symptomatologie anxieuse. Cela tend à montrer qu'avant de travailler sur les cognitions, ce qui est difficile chez les enfants jeunes ou les personnes avec une démence ou une déficience intellectuelle, il est possible d'agir sur ces cognitions en modifiant le comportement ouvert par le biais des techniques classiques issues des théories de l'apprentissage [12].

Une fois le comportement cible présent, les gains pour l'enfant (climat familial apaisé, autonomie augmentée, valorisation par l'entourage social) ont permis le maintien du comportement sans renforçateur au bout de quelques semaines et la généralisation de celui-ci aux toilettes hors du domicile. Les énoncés de type «c'est plus facile debout ! » ont fait place à des énoncés de type « tu as vu j’ai fait assise ! ».

Notre cas s'apparente à celui de Boudoukha [1] par 1) la définition du trouble décrit (la dyschésie psychologique) 2) la présence d'une problématique anxieuse et 3) l'utilisation des principes des théories de l'apprentissage dans la thérapie ; il s'en distingue par 1) le fait qu'il n'y a pas eu de réapprentissage de l'exonération du point de vue musculaire et 2) l'utilisation de renforçateurs tangibles et alimentaires.

Compte-tenu de l'abondance de littérature sur les troubles sphinctériens chez l'enfant, des propositions qui ont été faites récemment de consultations dédiées [15], mais également de 
l'utilité des stratégies fondées sur la modification du comportement $[6,16]$, des études de cas systématisées pourraient permettre de développer des lignes de conduites utiles aux cliniciens, pédiatres, pédopsychiatres, psychologues, médecins généralistes, sollicités par les familles.

\section{Conclusion}

La dyschésie psychologique de l'enfant proposée récemment par Boudoukha [1] comme nouvelle forme de dyschésie, est un outil pertinent en clinique comportementale et cognitive, à la fois parce qu'elle recouvre une réalité rencontrée par les cliniciens et qu'elle devrait permettre de formaliser un accompagnement de l'enfant et de sa famille dans un protocole se fondant sur les principes des théories de l'apprentissage, ce qui serait particulièrement utile pour les cliniciens.

\section{Déclaration d'intérêts}

C.C. : la jeune patiente est un membre de la famille de l'auteure

\section{Références}

1. Boudoukha AH. La dyschésie psychologique de l'enfant : anxiété, éducation comportementale et TCC. J Ther Comport Cogn 2013;23:124-31.

2. APA. DSM-IV : Text Revision. Manuel diagnostique et statistique des troubles mentaux. Paris: Masson; 2004.

3. Heymen S. Psychological and cognitive variables affecting treatment outcomes for urinary and fecal incontinence. Gastroenterol 2004;126(Suppl. 1):S146—51.

4. Lawless MR, McElderry DH. Noctural Enuresis: Current Concepts. Pediatr Rev 2001;22(12):399-407.

5. Kalach N, Campeotto F, Arhan P, Benhamoub P-H, Dupont C. Constipation fonctionnelle de l'enfant : stratégie des explorations et orientations. J Pediatr Pueric. 2009;22(7-8):326-36.

6. Hyman PE, Cocjin J, Oller M. Infant dyschezia. Clin Pediatr (Phila) 2009;48(4):438-9.

7. Juguet F, Siproudhis L. La dyschésie. Act Med Int Gastroenterol 1998;12(3):97-100.

8. Staumont G. Diagnostic et traitement d'une dyschésie. Gastroenterol Clin Biol 2006;30(3):427-38.

9. Samuel Nurko S, Di Lorenzo C. Functional Abdominal Pain: Time to Get Together and Move Forward. J Pediatr Gastroenterol Nutr 2008;47:679-715. 
10. Van der Plas R, Benninga M, Büller H, Bossuyt P, Akkermans L, Redekop W, et al. Biofeedback training in treatment of childhood constipation: a randomised controlled study. Lancet 1996;348(9030):776-8.

11. Clément C. Apprentissage, Conditionnement et Comportements Humains: Dunod; 2013.

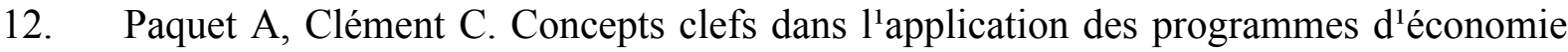
de jetons en institution $\mathrm{d}^{1}$ accueil et de soin pour $\mathrm{l}^{1}$ enfant et $\mathrm{l}^{1}$ adolescent. Rev Francoph Clin Comport Cogn 2008;XIII(3):25-31.

13. Simon JL, Thompson RH. The effects of undergarment type on the urinary contingence of toddlers. J Appl Behav Anal 2006;39(3):363-8.

14. Timelli I, Gautheron M, Gautheron V, Charles R. Encoprésie et constipation chez l'enfant. Deuxième partie : place du dispositif hospitalier dans cette pathologie. Medecine 2013;9(4):169-71.

15. Brazzelli M, Peter V Griffiths PV, Cody JD, Tappin D. Behavioural and cognitive interventions with or without other treatments for the management of faecal incontinence in children. Cochrane Database of Systematic Reviews 2011, Issue 12. Art. No: CD002240. DOI: 10.1002/14651858.CD002240.pub4. 